This article was downloaded by: [University of Arizona]

On: 10 January 2013, At: 04:09

Publisher: Taylor \& Francis

Informa Ltd Registered in England and Wales Registered Number: 1072954 Registered office: Mortimer House, 37-41 Mortimer Street, London W1T 3J H, UK

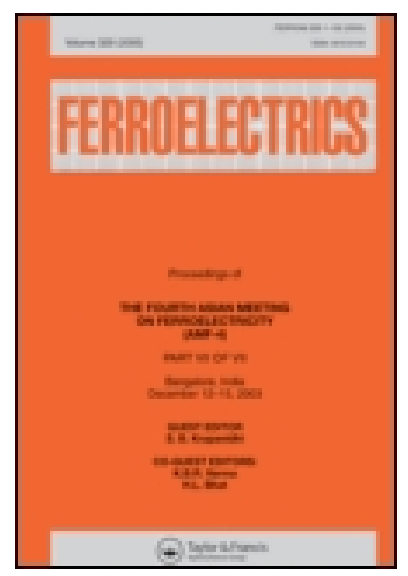

\title{
Ferroelectrics
}

Publication details, including instructions for authors and subscription information:

http:// www. tandfonline.com/loi/gfer20

\section{Diagrams of State for One-Dimensional Hydrogen-Bonded Proton Conductor}

\author{
I. V. Stasyuk ${ }^{a}, 0$. Vorobyov ${ }^{a} \&$ R. Ya. Stetsiv ${ }^{a}$ \\ a Institute for Condensed Matter Physics of the National Academy of \\ Sciences of Ukraine, 1 Svientsitskii str., 79011, Lviv, Ukraine
}

To cite this article: I. V. Stasyuk, O. Vorobyov \& R. Ya. Stetsiv (2012): Diagrams of State for OneDimensional Hydrogen-Bonded Proton Conductor, Ferroelectrics, 426:1, 6-12

To link to this article: http:// dx.doi.org/ 10.1080/00150193.2012.671087

\section{PLEASE SCROLL DOWN FOR ARTICLE}

Full terms and conditions of use: http://www.tandfonline.com/page/terms-and-conditions

This article may be used for research, teaching, and private study purposes. Any substantial or systematic reproduction, redistribution, reselling, loan, sub-licensing, systematic supply, or distribution in any form to anyone is expressly forbidden.

The publisher does not give any warranty express or implied or make any representation that the contents will be complete or accurate or up to date. The accuracy of any instructions, formulae, and drug doses should be independently verified with primary sources. The publisher shall not be liable for any loss, actions, claims, proceedings, demand, or costs or damages whatsoever or howsoever caused arising directly or indirectly in connection with or arising out of the use of this material. 


\title{
Diagrams of State for One-Dimensional Hydrogen-Bonded Proton Conductor
}

\author{
I. V. STASYUK, ${ }^{*}$ O. VOROBYOV, AND R.YA. STETSIV \\ Institute for Condensed Matter Physics of the National Academy of Sciences \\ of Ukraine, 1 Svientsitskii str., 79011 Lviv, Ukraine
}

\begin{abstract}
We investigate the equilibrium states of one-dimensional proton conductor where protons can move in the subsystem of hydrogen bonds. The consideration is based on the orientational-tunnelling model that takes into account two-stage mechanism of proton transfer (inter-bond and intra-bond hopping according to Grotthuss) as well as the short-range interaction between protons. Using exact diagonalization method for the finite cluster we have obtained one-particle spectral densities and found conditions of appearance of the various states of the system, in particular, charge density wave $(C D W)$ like state and the state similar to superfluid $(S F)$ phase (that can be analogues to superionic one). The corresponding diagrams of states are built and discussed.
\end{abstract}

Keywords Proton conductors; hydrogen bond; correlation; superionic transition

\section{Introduction}

In recent decades the systems with ionic and proton conductivity are getting more and more popular. Though solid state ionic conductors are known for almost two hundred years the intensive study of these objects were induced by the discovery of superionic (superprotonic) conductors. The latter are very promising from the point of view of their applications that include solid electrolyte batteries, fuel cells etc [1]. The special attention is paid to the crystals with hydrogen bonds that exhibit transition to superprotonic (superionic) phase with high conductivity that arises due to the motion of protons. Extensive experimental studies of these systems (see [2] for a review) revealed that the charge transfer occurs within the network of hydrogen bonds that connect ionic groups and form, in particular, quasi-one-dimensional (chain-like) [3-5] structures. For various crystals it was shown that superionic phase transition is driven by the transformation in proton subsystem (see [2] for review).

Though physical properties of superionic (superprotonic) conductors were thoroughly studied experimentally, the investigation of the microscopic mechanism of phase transitions was done within very simple and approximate theoretical approaches. Several variations of lattice gas model were used for this purpose. Some of these approaches were focused on long-range interaction [6, 7] and treated superionic transition as order-disorder one. These authors considered the network of virtual positions that can be occupied by ions (or virtual

Received in final form September 25, 2011.

*Corresponding author. E-mail: ista@icmp.lviv.ua 
hydrogen bonds that can be occupied by protons). In superionic (superprotonic) phase these positions are occupied partially with the same probability. The other authors have studied the influence of phonons on the proton subsystem and the transition from ferroelastic to superionic phase [8-10]. Some recent attempts based on the orientational-tunnelling model [11] have developed the approach focused on the short-range interaction between protons while also including the proton hopping into consideration [12-14].

All of the above-mentioned lattice models have treated protons in frames of Fermi statistics. However more correct consideration of protons (and ions in general) should be based on the mixed statistics of Pauli [15] since these particles are actually bosons but they occupy localized positions according to Fermi rule. Though the use of Pauli operators generate some additional mathematical complexities this approach opens up new possibilities. For instance, the lattice model of ionic Pauli conductor is capable to describe the appearance of superfluid-like state (that may correspond to superionic phase) in the system even in the absence of interaction between particles [16, 17]. While turning on interaction allows to describe more complex behaviour including transition to CDWlike state [18]. The lattice model of Pauli particles is similar to hard-core Bose-Hubbard model widely used for the description of physical phenomena in optical lattices as well as ionic conductivity and intercalation phenomena [19]. Bose-Hubbard model also exhibits the transition from the so-called Mott insulator state to superfluid-like state (see [20] for review). Some of the authors also mention the possibility of formation of intermediate "supersolid" phase that may appear on the phase diagrams alongside the transition from dielectric (CDW) to superfluid phase.

Here we consider the one-dimensional Pauli (hard-core boson) lattice model of proton conductor taking into account both proton transfer (according to two-stage Grotthuss mechanism) and short-range interaction between protons. We pay special attention to the latter ones since according to experiments [21] and quantum-chemical calculations [22, 23] they can be significant in real systems and therefore may affect the system's behaviour considerably. Moreover, for the case of ionic Pauli conductor the short-range interactions are responsible for the transition to CDW-like state [18]. Analyzing the single-particle spectral densities of protons in the finite 1D hydrogen-bonded chain and their changes with temperature at different concentration of protons we obtain the diagrams of state. The conditions of the crossover-type transition from CDW-like state to the superfluid(SF)-like state (that can be considered as an analogue of the superionic phase) and to the state that is similar to the Mott-insulator phase are discussed.

\section{The Model}

We use hard-core boson lattice model to describe proton conductor. From one hand such a model is similar to the ones used for strongly-correlated electron systems (i.e. the Hubbard model), but from the other hand it takes into account the specific feature of the object it is applied to. Particularly, the two-stage Grotthuss mechanism of the proton transport is taken into account via two proton transfer constants $\left(\Omega_{0}\right.$ for intra-bond hopping between two possible positions $a$ and $b$ on each bond as well as $\Omega_{R}$ for inter-bond transfer between hydrogen bonds that arises due to orientational motion of ionic groups) and because of that the model is known as orientational-tunnelling model [11]. It also includes the correlation between nearest protons caused by the short-range repulsion (the corresponding energies $U, V$ on the bond and $\left.w, w^{\prime}, \varepsilon\right)$. Here we introduce effective correlation parameters $V_{e f}=$ $U+V$ and $w_{e f}=w+w^{\prime}-2 \varepsilon$ that allows us to rewrite the Hamiltonian proposed in [11] 

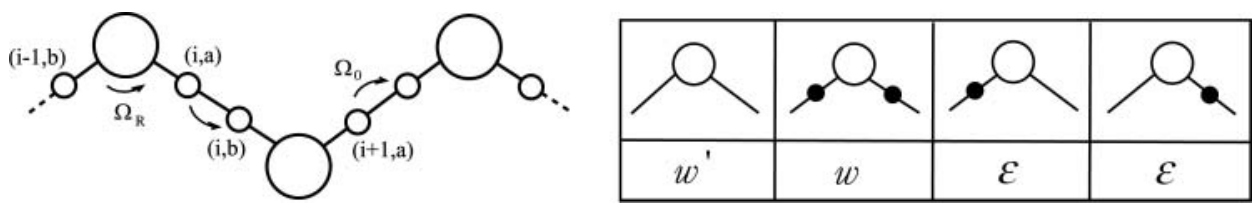

Figure 1. The model of one-dimensional hydrogen-bonded proton conductor. The large circles are ionic groups connected with the lines of hydrogen bonds. The small circles denote two possible positions of proton on each hydrogen bond. The table illustrates the energies of different configuration of protons near ionic group.

as follows

$$
\begin{aligned}
H & =(\varepsilon-\mu) \sum_{i}\left(n_{i a}+n_{i b}\right)+V_{e f} \sum_{i} n_{i a} n_{i b}+w_{e f} \sum_{i} n_{i b} n_{i+1, a} \\
& +\Omega_{0} \sum_{i}\left(c_{i a}^{+} c_{i b}+c_{i b}^{+} c_{i a}\right)+\Omega_{R} \sum_{i}\left(c_{i b}^{+} c_{i+1, a}+c_{i+1, a}^{+} c_{i b}\right) .
\end{aligned}
$$

In contrast to [11] $c_{i, \alpha}\left(c_{i, \alpha}^{+}\right)$in our case are Pauli operators. They describe the process of annihilation (creation) of proton in position $i, \alpha(\alpha=a, b)$ therefore $n_{i, \alpha}=c_{i, \alpha}^{+} c_{i, \alpha}$ is the occupation number of protons in this position (see Fig. 1).

\section{Exact Diagonalization Technique}

We calculate the single-particle spectral densities of one-dimensional proton Pauli conductor using exact diagonalization technique. For the finite chain of $N$ hydrogen bonds we introduce the many-particle states

$$
\left|n_{1, a} n_{1, b} \ldots n_{N, a} n_{N, b}\right\rangle ; \quad n_{i, \alpha}=0,1
$$

The Hamiltonian matrix on the basis of these states is the matrix of the order $2^{2 N} \times 2^{2 N}$.

This matrix is diagonalized numerically

$$
U^{-1} H U=\widetilde{H}=\sum_{p} \lambda_{p}|p\rangle\langle q|,
$$

where $\lambda_{p}$ and $|p\rangle$ are eigenvalues and eigenvectors of the Hamiltonian. The same transformation is applied to the creation and annihilation operators

$$
U^{-1} c_{i, \alpha} U=\sum_{p q} A_{p q}|p\rangle\left\langle q\left|, \quad U^{-1} c_{i, \alpha}^{+} U=\sum_{p q} A_{r s}^{*}\right| r\right\rangle\langle s|
$$

which are required to construct the two-time retarded Green's function $\ll c_{i, a} \mid c_{i, a}^{+} \gg$ that contains information about the one-particle energy spectrum of the system. For Pauli creation and annihilation operators this Green's function can be constructed in two ways, i.e. commutator Green's function

$$
\ll c_{i, \alpha}(t) \mid c_{i, \alpha}^{+}\left(t^{\prime}\right) \gg=-i \Theta\left(t-t^{\prime}\right)\left\langle\left[c_{i, \alpha}(t), c_{i, \alpha}^{+}\left(t^{\prime}\right)\right]\right\rangle
$$


or anticommutator Green's function

$$
\ll c_{i, \alpha}(t) \mid c_{i, \alpha}^{+}\left(t^{\prime}\right) \gg=-i \Theta\left(t-t^{\prime}\right)\left\langle\left\{c_{i, \alpha}(t), c_{i, \alpha}^{+}\left(t^{\prime}\right)\right\}\right\rangle .
$$

Imaginary parts of these Green's functions are the single-particle spectral densities (also referred to as densities of states or DOS)

$$
\begin{aligned}
\rho(\omega) & =-\frac{1}{\pi N} \sum_{i=1}^{N} \operatorname{Im}\left\langle c_{i, a} \mid c_{i, a}^{+}\right\rangle \\
& =-\frac{1}{\pi N} \sum_{i=1}^{N} \operatorname{Im}\left[\frac{1}{Z} \sum_{p q} A_{p q} A_{p q}^{*} \frac{e^{-\beta \lambda_{p}}-\eta e^{-\beta \lambda_{q}}}{\omega-\left(\lambda_{q}-\lambda_{p}\right)}\right],
\end{aligned}
$$

where $Z=\sum_{p} e^{-\beta \lambda_{p}}$. Spectral densities in (7), obtained from commutator $\eta=1$ (5) and anticommutator $\eta=-1$ (6) Green's functions respectively, exhibit discrete structure, i.e. they consist of several $\delta$-peaks due to the finite size of a cluster. Therefore we introduce small parameter $\Delta$ to broaden the $\delta$-peaks according to Lorentz distribution $\delta(\omega) \rightarrow \frac{1}{\pi} \frac{\Delta}{\omega^{2}+\Delta^{2}}$. Throughout our calculation $\Delta=0.25$. To eliminate the effect of boundaries, we apply the periodic boundary conditions to the cluster of $N$ hydrogen bonds. In numerical calculations we take the value $N=5$ that corresponds to 10 virtual proton positions.

\section{Proton Spectral Densities and Diagrams of State}

We have calculated the spectral densities (7) for a wide range of correlation strength, temperatures and values of chemical potential. The average concentration $\langle n\rangle$ at given $\mu$ was calculated according to spectral theorem $\langle n\rangle=\int_{-\infty}^{\infty} \frac{\rho(\omega) \mathrm{d} \omega}{\mathrm{e}^{\beta \omega}+1}$. Analyzing the changes of shape and character of frequency-depended spectral densities we have built the corresponding diagrams of state (Fig. 2).

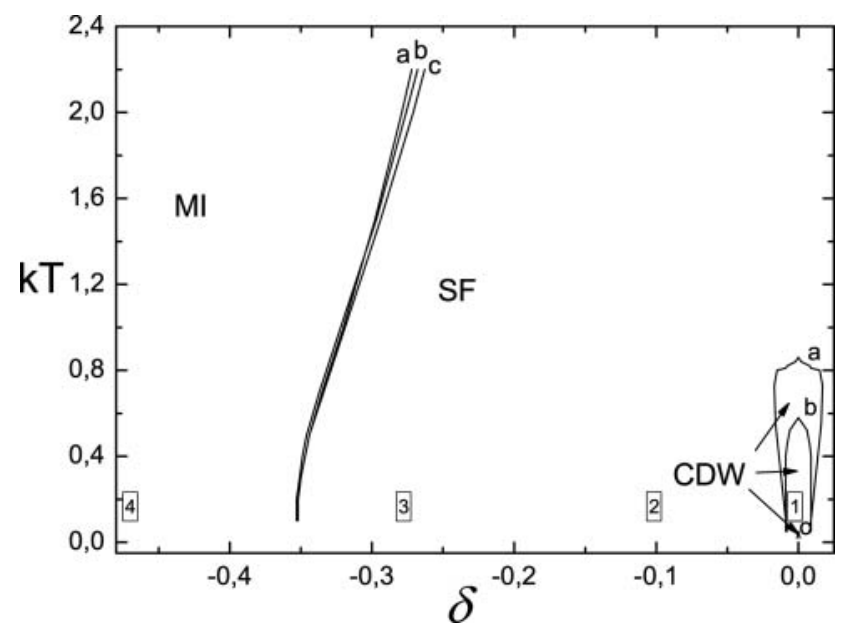

Figure 2. The diagram of state for different correlation parameters a) $V_{e f}=6, w_{e f}=4$; b) $V_{e f}=$ $6, w_{e f}=2 ;$ c) $V_{e f}=4, w_{e f}=2.8 . \delta=<n>-1 / 2$ denotes the deviation from half-filling. [, [2, [3, 4 denote the points on the diagram illustrated with the corresponding spectral densities on Fig. 3 
We have used the fact that in the SF phase the characteristic feature of the commutator spectral density is the existence of the negative branch (at $\omega<0)$ which appears continuously in $\omega=0$ point (see, for example [24]). Contrary to this, in the CDW phase this branch is separated from the positive one by the gap. The latter also exists in the MI phase but it has another meaning as it indicates the distance between the level of chemical potential and the edge of one-particle proton energy band (the negative branch is absent in this case).

In numerical calculations we have widely varied the values of short-range correlation constants relatively to transfer parameters. Experimental data and quantum-chemical calculations as well as semi-empirical theoretical estimations propose a wide range of values for interaction constants depending on the object considered. While transfer parameters are estimated as $\Omega_{0}=40-250 \mathrm{~cm}^{-1}, \Omega_{R}=200-1000 \mathrm{~cm}^{-1}$, interaction strengths in usual hydrogen-bonded superionic conductors can be by nearly an order of magnitude greater $[8,9]$. On the other hand, in molecular systems the latter can reach quite large values $V_{e f} \approx 6 \cdot 10^{3} \mathrm{~cm}^{-1}, w_{e f} \approx 10^{3}-10^{4} \mathrm{~cm}^{-1}[21,22]$. Below we present results obtained here for the cases $V_{e f} / \Omega_{0} \sim 4-6, w_{e f} / \Omega_{0} \sim 2-4, \Omega_{R} / \Omega_{0}=1.5$ (accordingly, on figures all energy parameters including $k T$ are presented in relation to $\Omega_{0}$ which is treated as an energy unit).

Let us first analyze the proton spectra (particularly, commutator spectral density) near half-filling (one proton per hydrogen bond). The case of strongly correlated proton conductor $\left(V, w>\sim \Omega_{0}, \Omega_{R}\right)$ corresponds to the gapped spectra that describes CDW-like state (Fig. 3.1). In this case the level of the chemical potential is near the middle of the gap $(\langle n\rangle \sim 1 / 2)$ and one may observe the situation when all protons occupy $a$ positions (or all protons occupy $b$ positions) along the chain. Due to the simplicity of the model this situation corresponds to ferroelectric type ordering, however it has more general meaning. The protons only occupy some of the positions available (while other positions remain unoccupied) that is a general feature of ordered phases that exist in superionic crystals. Similar effect was found for ionic and proton conductors described by the analogous models in frames of Fermi statistics [13,14] as well as for the Pauli ionic conductor [18]. Keeping this in mind we will call this state CDW though the doubling of lattice period is not observed (while it is observed in "real" CDW phases). For the case of ionic conductor (both Pauli and Fermi) the splitting of spectra occurred due to charge ordering with doubling of lattice period. In the CDW-state the conductivity is weak as it may arise only due to the motion of D- and L-defects while the energy of forming of pair of these defects is about the width of the gap. At weaker interactions $\left(V, w \sim \Omega_{0}, \Omega_{R}\right)$ as well as at higher temperatures the gap that exists in the proton spectra near half-filling may vanish and the system undergoes the transition to SF-state. This transition can be also achieved by the change of the chemical potential or conjugated change of average concentration (Figs. 3.2 and 3.3). The conductivity in this state increases dramatically and we can match it with the experimentally observed superionic phase. Moreover as we go far away from half filling (or considerably decrease $\mu$ ) we obtain another transition to the state that has no negative branch (Fig. 3.4). The spectrum in this case is gapless but the level of the chemical potential is below the band. This state is interpreted as Mott insulator (MI) state because the protons need some activation energy to induce their transport. The situation is similar to the one observed, in particular, in optical lattices in the vicinity of SF-MI transition at intermediate concentrations. However for more thorough description of the proton subsystem in this case one would have to include the long-range interactions into consideration. At low temperatures the latter induce the real CDW state accompanied by charge modulation 

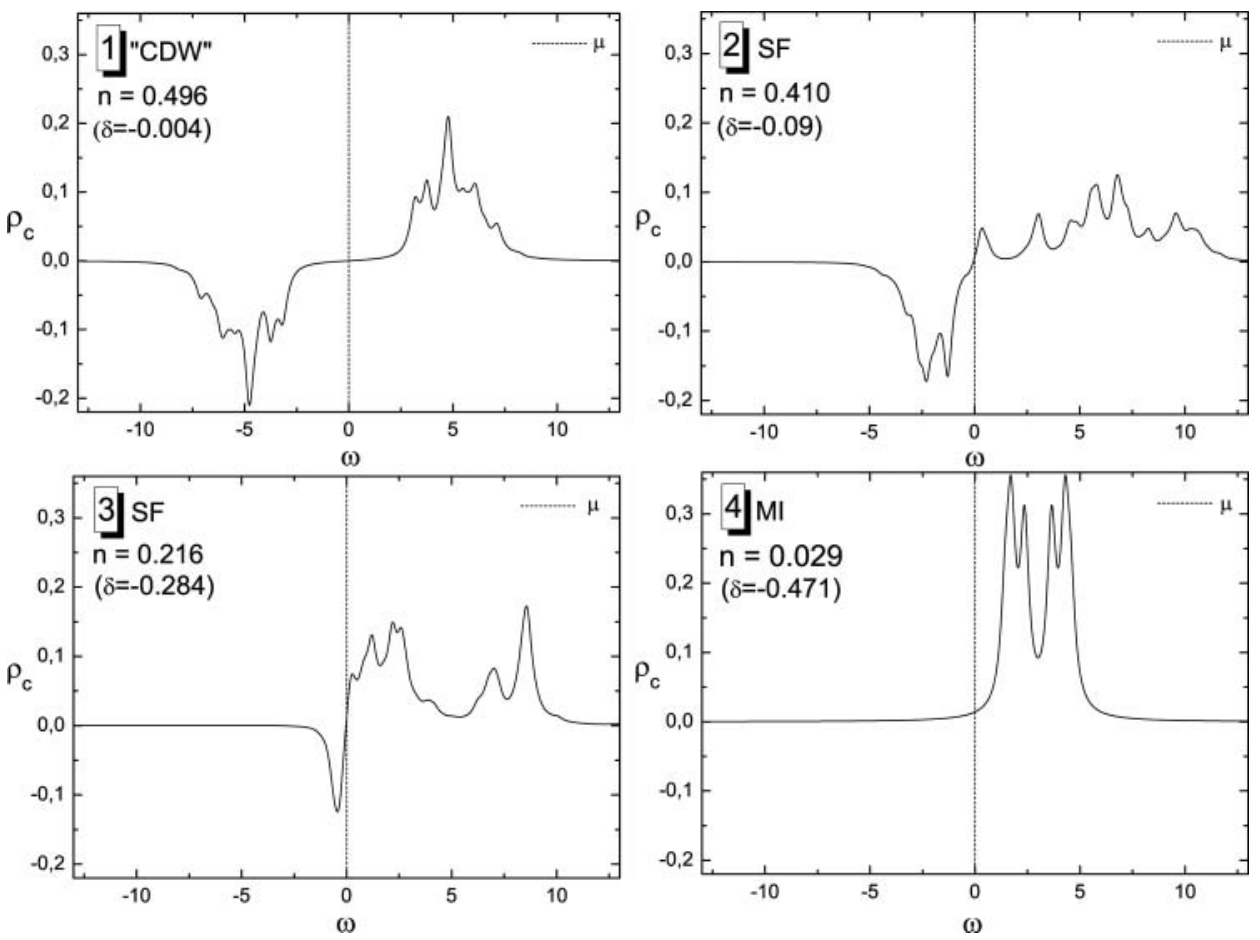

Figure 3. The commutator spectral densities that correspond to CDW, SF and MI states. $V_{e f}=$ $6, w_{e f}=4, k T=0.2$. The dashed curve denotes the level of the chemical potential.

when some of the virtual hydrogen bonds in the unit cell are occupied. This kind of state would be an analogue of ferroelastic phases in real superionic conductors.

Figure 4 presents the diagrams of state $\left(T, w_{e f}\right),\left(w_{e f}, \delta\right)$ in the vicinity of half-filling (1 proton per bond) where we observe transition from charge ordered state (CDW) to SF-state both at increase of temperature and decrease of interaction. As we go away from half filling $(\delta \neq 0)$ the critical value of correlation $w_{e f}$ that separates SF and CDW states
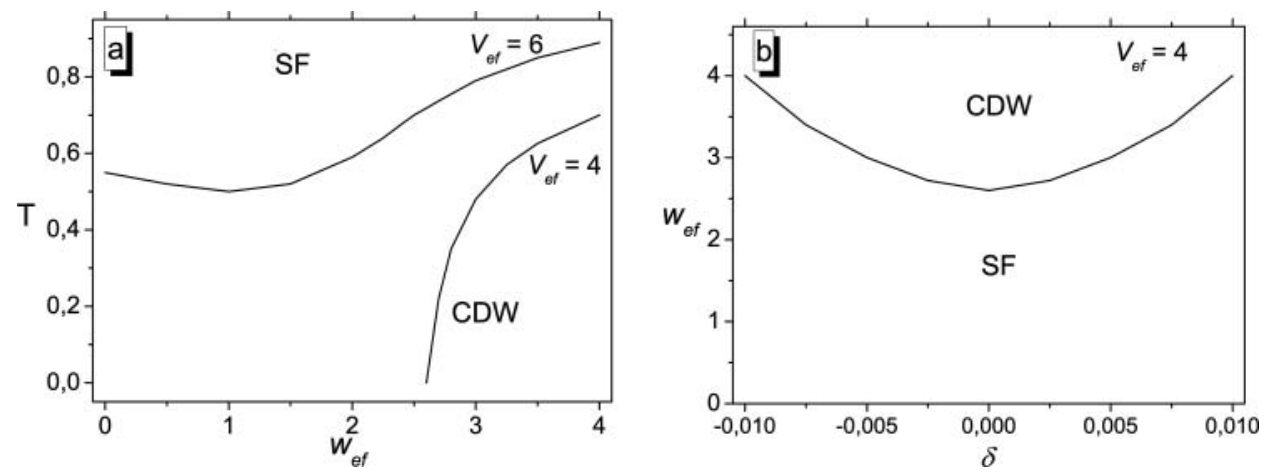

Figure 4. The diagrams of states. a). ( $\left.T, w_{e f}\right)$ diagram for different values of interaction $\left.V_{e f} ; \mathrm{b}\right)$. $\left(w_{e f}, \delta\right)$ diagram for $V_{e f}=4, T=0.05$. 
increases. This result is in agreement with the ones obtained for hard-core boson with pair interaction via Monte-Carlo simulations and within mean-field approach [25]. In $T \rightarrow 0$ limit the transition between this states becomes a quantum phase transition.

\section{Conclusions}

Calculating the spectral densities for hydrogen-bonded one-dimensional proton conductor described by Pauli orientational-tunnelling model we have found the regions where various equilibrium states (phases) exist. We have found that at relatively strong correlations and low temperatures the charge ordered state (CDW-like) in this system is observed near half-filling. This state is an analogue of ferroelastic phase of superionic conductors. As we increase temperature the system undergoes the crossover transition to SF-state that can be considered as superionic phase. Clearly, this one-dimensional model is too simple to fully describe this kind of transitions in three-dimensional crystals, nevertheless one can easily fit the values of ratios $V_{e f} / \Omega_{0}, w_{e f} / \Omega_{0}$ to obtain the experimentally observed superionic transition temperatures of several hundred $\mathrm{K}$.

\section{References}

1. K. D. Kreuer, Phys. Chem. Chem. Phys. 3, 771 (2002).

2. Superionic conductor physics: Proceedings of the 1st International Discussion Meeting, ed. J. Kawamura et al., 2007, World Scientific, 204 p.

3. A. V. Belushkin, V. I. F. David, R. M. Ibberson, and L. A. Shuvalov, Acta Cryst. B 47, 161 (1991).

4. T. Pawłowski, M. Połomska, B. Hilczer, and L. Kirpichnikova, J. Mol. Struct. 555, 317 (2000).

5. D. Arčon, R. Blinc, J. Dolinšek, L. and A. Shuvalov, Phys. Rev. B 55, 8961 (1997).

6. W. Salejda and N. A. Dzhavadov, Phys. Stat. Sol. (b) 158, 475 (1990).

7. I. V. Stasyuk, N. Pavlenko, and B. Hilczer, Phase Transitions 62, 135 (1997).

8. N. I. Pavlenko, Phys. Stat. Sol. (b) 218, 295 (2000).

9. N. I. Pavlenko and I. V. Stasyuk, J. Chem. Phys. 114, 4607 (2001).

10. V. V. Krasnogolovets and P. M. Tomchuk, Phys. Stat. Sol. (b) 130, 807 (1985).

11. I. V. Stasyuk, O. L. Ivankiv, and N. I. Pavlenko, J. Phys. Studies 1, 418 (1997).

12. I. Stasyuk, O. Vorobyov, and B. Hilczer, Solid Sate Ionics 145, 211 (2001).

13. I. V. Stasyuk and O. Vorobyov, Phase Transitions 80, 63 (2007).

14. I. V. Stasyuk and O. Vorobyov, Ferroelectrics 376, 64 (2008).

15. G. D. Mahan, Phys. Rev. B 14, 780 (1976).

16. I. V. Stasyuk and I. R. Dulepa, J. Phys. Studies 13, 2701 (2009).

17. R. Micnas, J. Ranninger, and S. Robaszkiewicz, Rev. Mod. Phys. 62, 170 (1990).

18. I.V. Stasyuk, O. Vorobyov, and R. Ya. Stetsiv, Condens. Matter Phys. 14, 23702 (2011).

19. T. S. Mysakovych, V. O. Krasnov, and I. V. Stasyuk, Condens. Matter Phys. 11, 663 (2008).

20. I. Bloch, J. Dalibard, and W. Zwerger, Rev. Mod. Phys. 80, 885 (2008).

21. R. Hassan and E. S. Campbell, J. Chem. Phys., 1992, 97, 4362.

22. M. Eckert and G. Zundel, J. Phys. Chem., 1988, 92, 7016.

23. I. V. Stasyuk, R.Ya. Stetsiv, and Yu.V. Sizonenko, Condens. Matter Phys. 5, 685 (2002).

24. C. Menotti and N. Trivedi, Phys. Rev. B 77, 235120 (2008).

25. G. Schmid, S. Todo, M. Troyer, and A. Dorneich, Phys. Rev. Lett. 88, 167208 (2002). 